\title{
BMJ Open Association between serum $\gamma$-glutamyltranspeptidase and atherosclerosis: a population-based cross-sectional study
}

\author{
Takuya Fukuda, ${ }^{1}$ Masahide Hamaguchi, ${ }^{1}$ Takao Kojima, ${ }^{2}$ Yasuhiro Ohshima, ${ }^{2}$ \\ Akihiro Ohbora, ${ }^{2}$ Takahiro Kato, ${ }^{2}$ Naoto Nakamura, ${ }^{1}$ Michiaki Fukui ${ }^{1}$
}

To cite: Fukuda T, Hamaguchi M, Kojima T, et al. Association between serum $\gamma$-glutamyltranspeptidase and atherosclerosis: a population-based crosssectional study. BMJ Open 2014;4:e005413.

doi:10.1136/bmjopen-2014005413

- Prepublication history for this paper is available online. To view these files please visit the journal online (http://dx.doi.org/10.1136/ bmjopen-2014-005413).

Received 6 April 2014

Revised 29 August 2014

Accepted 2 September 2014

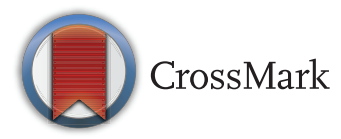

${ }^{1}$ Department of

Endocrinology and

Metabolism, Kyoto

Prefectural University of

Medicine, Graduate School of

Medical Science, Kyoto,

Japan

${ }^{2}$ Department of

Gastroenterology, Murakami

Memorial Hospital, Asahi

University, Gifu, Japan

Correspondence to Dr Masahide Hamaguchi; seele@koto.kpu-m.ac.jp

\section{ABSTRACT}

Objective: The purpose of this study was to determine the relationship between serum $\gamma$ glutamyltranspeptidase (GGT) and brachial-ankle pulse wave velocity (baPWV) as an indicator for atherosclerosis in Japanese men and women after adjusting for fatty liver.

Design: A cross-sectional study.

Setting: A health check-up centre in Japan. Participants: 912 Japanese men and women aged 24-84 years recruited from people who received a medical health check-up programme with a standardised questionnaire and an automatic waveform analyser to measure baPWV.

Main outcome measures: We measured serum GGT concentrations and baPWV. Fatty liver was diagnosed by standardised criteria using abdominal ultrasonography. The postmenopausal state was defined as beginning 1 year after the cessation of menses.

Results: In women, $\log _{2}$ GGT was positively associated with baPWV ( $\beta=0.11,95 \% \mathrm{Cl} 0.02$ to 0.19 , $p<0.05$ ), independent of age, body mass index, systolic blood pressure, fasting plasma glucose, triglycerides, estimated glomerular filtration rate, fatty liver, menopausal state and parameters of lifestyles. However, in men, the positive association of $\log _{2}$ GGT with baPWV was not significant $(\beta=-0.04,95 \% \mathrm{Cl}$ -0.10 to $0.03, p=0.28$ ) in multivariable linear regression analyses.

Conclusions: The serum GGT level was associated with baPWV, independently of covariates including fatty liver or menopausal state just in women, but not in men.

\section{INTRODUCTION}

Serum $\gamma$-glutamyltranspeptidase (GGT) is a glycoprotein with a molecular weight of $68000 \mathrm{Da}$, consisting of two proteins, the larger chain with a molecular weight of $46000 \mathrm{Da}$ and the smaller one with a molecular weight of $22000 \mathrm{Da}^{1}{ }^{1}$ There is a

\section{Strengths and limitations of this study}

- This is a large population-based, cross-sectional study in which we used a standardised questionnaire for lifestyle factors, so our data are accurate and the classification is systematic.

- This study shows a difference in the relationship between $\log _{2}$ GGT and baPWV depending on sex.

- We applied multivariable linear regression analyses with three models to examine the relationship of $\log _{2}$ GGT and baPWV, and confirmed the solidity of the results independent of the selection of covariates.

- The menopausal state was based on a self-report and we did not measure the serum oestradiol level.

- This is a cross-sectional study, so we cannot infer the causality.

membrane form of GGT as well as secreted GGT. Secreted GGT as well as membrane GGT acts as a protein catalyst in the degradation of glutathione, which is a major thiol antioxidant in the body. Thus, serum GGT has been regarded as a marker of oxidative stress. $^{2}$

In clinical practice, serum GGT is measured to evaluate the damage of alcoholic liver disease, cholestasis and biliary injury. ${ }^{3}$ The increased level of serum GGT is a risk factor for poor prognosis in patients with hepatocellular carcinoma or hepatobiliary disease. ${ }^{4}$ In addition, recent studies have reported the possibility that the increased level of serum GGT might be a risk factor for cardiovascular disease (CVD) mortality. ${ }^{5-7}$ Moreover, it was shown by analysis of the British Women's Heart and Health study and the meta-analysis of population-based studies that the hazard risk of natural logged GGT was 1.20 (95\% CI 1.02 to 1.40$)$ for a risk of CVD independent of alcohol consumption. ${ }^{8}$ 
Additionally, a strong association between increasing GGT levels and mortality from CVD was also reported in women. ${ }^{8}$ These studies indicated that the increased level of serum GGT could predict future events of CVD. However, the increased level of serum GGT was also observed in patients with fatty liver as well as women at the menopause state, except for participants who consume alcohol. Recently, fatty liver has been known as a risk factor of CVD in itself. ${ }^{9}$ Thus, it is unclear whether GGT is a risk factor for the future onset of CVD independent of fatty liver or not. Even if GGT is a risk factor for CVD independently, the mechanism underlying the increased level of serum GGT and CVD has not been well understood.

In fact, the association of the increased level of serum GGT with atherosclerosis seems controversial. In many cross-sectional studies, brachial-ankle pulse wave velocity (baPWV) has been broadly used as a marker of atherosclerosis. Although the increased level of serum GGT is reported to be associated with baPWV, it has been unclear whether the association between GGT and baPWV differs between men and women. In fact, three studies have reported that an increased level of GGT was associated with high baPWV in both sexes, ${ }^{10-12}$ but only one study has reported a positive association in men. ${ }^{13}$ Moreover, another study has reported the association only in women. ${ }^{14}$

Therefore, we performed a cross-sectional study to assess the sexual discrepancy in the relationship between GGT and baPWV as an indicator for atherosclerosis in the Japanese population.

\section{MATERIALS AND METHODS}

\section{Study design}

We performed a cross-sectional study involving participants of a medical health check-up programme including pulse wave velocity and abdominal ultrasonography. The programme was conducted in the Medical Health Checkup Center at Murakami Memorial Hospital, Gifu, Japan. The details of the medical health check-up programme were described previously. ${ }^{15}$ Briefly, the purpose of this programme is to promote public health through early detection of chronic diseases and the evaluation of their underlying risk factors. Known as a 'human dock', medical check-up services of this kind are very popular in Japan.

\section{Study population}

All individuals participating in such health check-up programmes including measurements of baPWV at Murakami Memorial Hospital between March 2004 and December 2012 were invited to join this study. The study was conducted in accordance with the Declaration of Helsinki. Informed consent was obtained from all participants.

Participants who received medication, including hormone replacement therapy and oral contraceptives, and tested positive for hepatitis B antigen and/or hepatitis $\mathrm{C}$ antibody, and pregnant women were excluded from the study. In addition, participants whose ankle-brachial index (ABI) was $<0.95$ were excluded to ensure the accuracy of baPWV measurements. ${ }^{16}$

\section{Measurement of baPWV}

We used an automatic waveform analyzer (Colin Medical Technology, Komaki, Japan) to measure baPWV and ABI by a previously reported method. ${ }^{17}$ This instrument measures pulse volumes of the brachial and posterior tibial arteries by an oscillometric method using cuffs adapted to both arms and ankles. The baPWV measurement was made in a room with a temperature around $25^{\circ} \mathrm{C}$ after the patient had rested for $5 \mathrm{~min}$ in a supine position. ECG electrodes were placed on both wrists, and a heart sound microphone was placed over the left edge of the sternal border. Cuffs were wrapped on the brachia and ankles and connected to a plethysmographic sensor that determines the volume pulse form and an oscillometric pressure sensor that measures blood pressure.

The path lengths from the suprasternal notch to the brachium (Lb) and from the suprasternal notch to the ankle (La) were calculated based on the participant's height. The delay time from the ascending point of the brachial waveform to the ascending point of each ankle waveform (DTba) was determined automatically. baPWV was calculated as the pulse wave propagation distance (Lb-La) divided by the pulse wave propagation time (DTba) and expressed in $\mathrm{cm} / \mathrm{s}$. The intraobserver and interobserver coefficients of variation were reported to be $10 \% \quad(\mathrm{r}=0.87, \mathrm{p}<0.01)$ and $8.4 \% \quad(\mathrm{r}=0.98, \mathrm{p}<0.01)$, respectively. ${ }^{17}$

\section{Data collection and measurements}

The health check-up programmes that were used for the collection of data included the following tests: urinalysis, blood cell counts, blood chemistry, measurements of hepatitis B antigen and hepatitis C antibody, ECG, chest radiography, barium examination of the upper gastrointestinal tract and abdominal ultrasonography. ${ }^{18}$

Blood samples were drawn from the antecubital vein of seated participants after $8 \mathrm{~h}$ of fasting. Samples were collected in siliconised glass tubes containing sodium fluoride for the glucose analysis and no additives for the serum. Plasma and serum samples were centrifuged immediately and stored at $-80^{\circ} \mathrm{C}$ until analysis. We undertook blood examinations with MODULAR ANALYTICS (Hitachi High-Technologies Corp. Ltd, Tokyo, Japan), which was broadly used for biochemical analysis in Japan. The $95 \%$ CI of measured GGT is $\pm 2.2 \mathrm{IU} / \mathrm{L}$ according to the manufacturer's data. The analytical coefficients of variation for GGT, aspartate transaminase, alanine aminotransferase, plasma glucose, triglycerides, high-density lipoprotein cholesterol, lowdensity lipoprotein cholesterol (LDL-C), uric acid and creatinine were $2 \%, 1.9 \%, 1.7 \%, 1.2 \%, 2.3 \%, 2.1 \%$, $2.5 \%, 0.9 \%$ and $1.4 \%$, respectively. 
The diagnosis of fatty liver was based on the results of abdominal ultrasonography, which was performed by trained technicians with Aloka SSD-650CL (Aloka Co, Ltd, Tokyo, Japan). All ultrasonographic images were stored as photocopies, and one gastroenterologist reviewed the images and made the diagnosis of fatty liver without reference to other individual data of any of the participants.$^{19}$ Of the four known criteria (hepatorenal echo contrast, liver brightness, deep attenuation and vascular blurring), the participants were required to have hepatorenal contrast and liver brightness to be given a diagnosis of fatty liver. ${ }^{20}$ Body mass index (BMI) was calculated as body weight in kilograms divided by the square of the participant's height in metres. Estimated glomerular filtration rate (eGFR) was calculated using the Japanese Society of Nephrology equation: eGFR $=194 \times \mathrm{Cr}^{-1.094} \times \mathrm{age}^{-0.287} \quad\left(\mathrm{~mL} / \mathrm{min} / 1.73 \mathrm{~m}^{2}\right)$ for men, and eGFR was multiplied by a correction factor of 0.739 for women. ${ }^{21}$

\section{Standardised questionnaire for lifestyle factors}

A standardised questionnaire was administered to all participants by the same trained team of interviewers. Habits regarding alcohol consumption were evaluated by asking the participants about the amount and type of alcoholic beverages consumed per week during the past month, then estimating the mean ethanol intake per week. The total amount of alcohol consumed per week was calculated in grams, and then categorised into the following four grades: non or minimal alcohol consumption, <40 g/week; light alcohol consumption, 40-140 g/week; moderate alcohol consumption, 140-280 $\mathrm{g} /$ week; and excess alcohol consumption, $>280 \mathrm{~g} /$ week. $^{19}{ }^{22}{ }^{23}$ Smoking status was also categorised into two groups (never smoker or ex-smoker and current smoker). ${ }^{19}$ On the questionnaire, participants reported the type, duration and frequency of their participation in sports or recreational activities. ${ }^{24}$ When participants performed any kind of sport regularly at least once a week, we categorised them as regular exercisers. ${ }^{25}$ The postmenopausal state was defined as beginning 1 year after the cessation of menses. ${ }^{26}$

\section{Statistical analysis}

The SPSS statistical package, V.11.0.1 J (SPSS, Inc, Chicago, Illinois, USA), was used for all statistical analyses and a $\mathrm{p}$ value less than 0.05 was considered statistically significant. Owing to the skewed distribution of GGT, data were normalised by logarithmic transformation for further statistical analysis. Also, since the standardised $\beta$ of GGT for baPWV has been controversial, a formal sample size estimate was not made a priori. Continuous values were expressed as mean $\pm \mathrm{SD}$ and categorical values were expressed as per cent (number). Two groups of participants were compared by using the unpaired $\mathrm{t}$ test and the $\chi^{2}$ test. The relationships of baPWV with logarithmic transformation of GGT $\left(\log _{2}\right.$
GGT), age, BMI, systolic blood pressure or other variables were examined by univariate linear regression analyses. We applied multivariable linear regression analyses to examine the relationship of $\log _{2}$ GGT and baPWV after adjusting covariates as follows-model 1: adjusted for age; model 2: with additional adjustment for BMI, systolic blood pressure and biochemical data; model 3 with adjustment for smoking states, physical activities, presence of fatty liver and menopausal state for women, which was defined as the primary outcome of the study. We selected age, BMI, fatty liver, menopausal state, parameters of lifestyles and significant variates in univariate linear regression analyses as covariates in the multivariable model.

\section{RESULTS}

\section{Participants}

We reviewed medical records with clinical characteristics of 1445 participants (897 men and 548 women) who participated in such health check-up programmes at Murakami Memorial Hospital between March 2004 and December 2012. We excluded 433 participants (284 men and 149 women) who were receiving medication, 26 (16 men and 10 women) who had positive hepatitis B antigen and/or positive antihepatitis $\mathrm{C}$ antibody, 66 women who received hormone replacement therapy, 1 woman who had oral contraceptives and 1 woman who was pregnant. Furthermore, we excluded 6 participants (5 men and 1 woman) whose ABI was $<0.95$. As a result, a total of 912 participants (592 men and 320 women) were included in the full analysis set (figure 1).

The clinical and laboratory characteristics of the 912 participants are presented in table 1 . The mean baPWV was $1411.5 \mathrm{~cm} / \mathrm{s}$ in men and $1363.0 \mathrm{~cm} / \mathrm{s}$ in women. The mean $\log _{2}$ GGT levels were $4.62 \mathrm{IU} / \mathrm{L}$ in men and $3.88 \mathrm{IU} / \mathrm{L}$ in women. One hundred and thirty-eight women $(43.1 \%)$ were at the premenopausal state and $182(56.9 \%)$ were at the postmenopausal state. The means of $\log _{2}$ GGT and baPWV were $3.78 \mathrm{IU} / \mathrm{L}$ in premenopausal versus $3.96 \mathrm{IU} / \mathrm{L}$ in postmenopausal women $(\mathrm{p}<0.01)$ and $1265.7 \pm 180.4 \mathrm{~cm} / \mathrm{s}$ in premenopausal versus $1473.1 \pm 247.8 \mathrm{~cm} / \mathrm{s}$ in postmenopausal women $(p<0.001)$, respectively. Interestingly, age was associated with $\log _{2}$ GGT after adjusting for fatty liver in

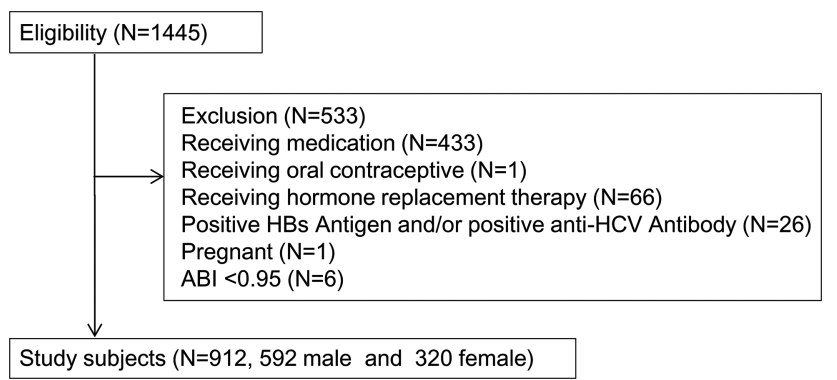

Figure 1 Study participants (ABI, ankle-brachial index; HBs, hepatitis B surface; HCV, hepatitis $C$ virus). 
Table 1 The clinical and laboratory characteristics of study participants

\begin{tabular}{|c|c|c|c|}
\hline & Males & Females & p Value \\
\hline $\mathrm{N}$ & 592 & 320 & \\
\hline baPWV (cm/s) & $1411.5 \pm 241.1$ & $1363.0 \pm 241.3$ & 0.10 \\
\hline $\mathrm{ABI}$ & $1.15 \pm 0.06$ & $1.12 \pm 0.06$ & 0.92 \\
\hline $\log _{2}$ GGT (IU/L) & $4.62 \pm 0.84$ & $3.88 \pm 0.63$ & $<0.001$ \\
\hline Age (years) & $50.5 \pm 9.8$ & $52.4 \pm 9.0$ & $<0.01$ \\
\hline BMI $\left(\mathrm{kg} / \mathrm{m}^{2}\right)$ & $23.7 \pm 3.1$ & $22.1 \pm 2.9$ & $<0.001$ \\
\hline Systolic blood pressure $(\mathrm{mm} \mathrm{Hg})$ & $122.9 \pm 14.4$ & $115.3 \pm 14.7$ & $<0.001$ \\
\hline Diastolic blood pressure (mm Hg) & $78.7 \pm 9.3$ & $71.4 \pm 9.5$ & $<0.001$ \\
\hline AST (IU/L) & $21.8 \pm 9.1$ & $19.2 \pm 5.5$ & $<0.001$ \\
\hline ALT (IU/L) & $25.8 \pm 16.0$ & $16.9 \pm 7.6$ & $<0.001$ \\
\hline Fasting glucose (mg/dL) & $99.7 \pm 11.5$ & $94.9 \pm 17.4$ & $<0.001$ \\
\hline Total cholesterol (mg/dL) & $207.1 \pm 34.8$ & $214.9 \pm 37.6$ & $<0.001$ \\
\hline Triglycerides (mg/dL) & $115.7 \pm 82.3$ & $70.6 \pm 46.6$ & $<0.001$ \\
\hline HDL-C (mg/dL) & $49.1 \pm 12.5$ & $61.8 \pm 14.6$ & $<0.001$ \\
\hline LDL-C (mg/dL) & $127.9 \pm 31.4$ & $128.4 \pm 32.2$ & 0.80 \\
\hline Uric acid (mg/dL) & $5.9 \pm 1.2$ & $4.1 \pm 0.9$ & $<0.001$ \\
\hline Serum creatinine (mg/dL) & $0.94 \pm 0.13$ & $0.68 \pm 0.10$ & $<0.001$ \\
\hline eGFR $\left(\mathrm{mL} / \mathrm{min} / 1.73 \mathrm{~m}^{2}\right)$ & $69.3 \pm 11.6$ & $72.5 \pm 12.5$ & $<0.001$ \\
\hline \multicolumn{4}{|l|}{ Grade of alcohol consumption } \\
\hline Non or minimal (0-40 g/week) (\% (n)) & $61.5(364)$ & $91.3(292)$ & $<0.001$ \\
\hline Light (40-140 g/week) (\% (n)) & $12.2(72)$ & $5.0(16)$ & $<0.001$ \\
\hline Moderate (140-280 g/week) (\% (n)) & $13.5(80)$ & $2.5(8)$ & $<0.001$ \\
\hline Heavy (more than $280 \mathrm{~g} /$ week) (\% (n)) & $12.8(76)$ & $1.2(4)$ & $<0.001$ \\
\hline \multicolumn{4}{|l|}{ Smoking } \\
\hline None or past $(\%(n))$ & $69.6(412)$ & $94.7(303)$ & $<0.001$ \\
\hline Current $(\%(n))$ & $30.4(180)$ & $5.3(17)$ & $<0.001$ \\
\hline \multicolumn{4}{|l|}{ Regular exerciser } \\
\hline$>1$ week (yes, \% (n)) & $18.8(110)$ & $21.6(67)$ & 0.31 \\
\hline \multicolumn{4}{|l|}{ Fatty liver } \\
\hline None $(\%(n))$ & $69.8(413)$ & $87.5(280)$ & $<0.001$ \\
\hline Moderate or severe $(\%(n))$ & $30.2(179)$ & $12.5(40)$ & $<0.001$ \\
\hline \multicolumn{4}{|l|}{ Menopausal status } \\
\hline Postmenopausal (\% (n)) & - & $56.9(182)$ & - \\
\hline
\end{tabular}

Data are shown as mean \pm SD.

$\mathrm{ABI}$, ankle brachial index; ALT, alanine aminotransferase; $\mathrm{AST}$, aspartate transaminase; baPWV, brachial-ankle pulse wave velocity; BMI, body mass index; eGFR, estimated glomerular filtration rate; GGT, $\gamma$-glutamyltranspeptidase; HDL-C, high-density lipoprotein cholesterol; LDL-C, low-density lipoprotein cholesterol.

women, but not in men. Because of possible interaction between GGT levels and oestrogen, we compared GGT levels and baPWV of women who received hormone replacement therapy and those of women who did not receive it. As a result, there were no differences between those groups.

\section{Main results}

To identify the covariates for the association of GGT with baPWV, we applied univariate linear regression analyses. In men, age, systolic blood pressure, diastolic blood pressure, fasting plasma glucose, triglyceride and eGFR were associated with baPWV (table 2). In women, age, systolic blood pressure, diastolic blood pressure, fasting plasma glucose, total cholesterol, triglycerides, LDL-C, eGFR, current smoker, fatty liver and postmenopausal state were associated with baPWV.

Therefore, we selected systolic blood pressure, fasting plasma glucose, triglyceride and eGFR as covariates of multivariable linear regression analyses, in addition to age, BMI, fatty liver, menopausal state and parameters of lifestyles. We selected age as the covariate in multivariable analysis model 1 . In model 1 , the age-adjusted model, standardised $\beta$ of $\log _{2}$ GGT for baPWV was 0.08 (95\% CI 0.01 to $0.15, \mathrm{p}<0.05)$ in men and 0.19 (95\% CI 0.10 to $0.28, \mathrm{p}<0.001$ ) in women (table 3 , model 1 ). We selected BMI, systolic blood pressure and biochemical data (fasting plasma glucose, triglycerides, eGFR) as well as age as covariates in model 2. In model 2, there was no significant association between baPWV and $\log _{2}$ GGT in men $(\beta=-0.03,95 \% \mathrm{CI}-0.09$ to $0.04, \mathrm{p}=0.43)$, although there was a significant association in women $(\beta=0.11$, $95 \%$ CI 0.03 to $0.20, \mathrm{p}<0.05$; model 2 ). At the final stage, we selected parameters of lifestyles (current smoker, regular exerciser), fatty liver and postmenopausal state as well as the aforementioned factors as covariates in model 3. Adjustment for the presence of parameters of lifestyles, fatty liver and postmenopausal 
Table 2 Correlations of clinical characteristics for baPWV in males and females

\begin{tabular}{|c|c|c|c|c|}
\hline & \multicolumn{2}{|l|}{ Males } & \multicolumn{2}{|l|}{ Females } \\
\hline & $\beta, 95 \% \mathrm{Cl}$ (low to high) & p Value & 及, 95\% Cl (low to high) & p Value \\
\hline $\log _{2}$ GGT (IU/L) & $0.10(0.02$ to 0.18$)$ & $<0.05$ & $0.31(0.20$ to 0.41$)$ & $<0.001$ \\
\hline Age (years) & $0.48(0.40$ to 0.55$)$ & $<0.001$ & $0.57(0.48$ to 0.66$)$ & $<0.001$ \\
\hline BMI $\left(\mathrm{kg} / \mathrm{m}^{2}\right)$ & $0.02(-0.06$ to 0.10$)$ & 0.63 & $0.10(-0.01$ to 0.21$)$ & 0.09 \\
\hline Systolic blood pressure $(\mathrm{mm} \mathrm{Hg})$ & $0.53(0.46$ to 0.60$)$ & $<0.001$ & $0.48(0.39$ to 0.58$)$ & $<0.001$ \\
\hline Diastolic blood pressure $(\mathrm{mm} \mathrm{Hg})$ & $0.48(0.41$ to 0.55$)$ & $<0.001$ & 0.43 (0.33 to 0.53$)$ & $<0.001$ \\
\hline Fasting plasma glucose (mg/dL) & $0.24(0.16$ to 0.31$)$ & $<0.001$ & $0.23(0.13$ to 0.34$)$ & $<0.001$ \\
\hline Total cholesterol (mg/dL) & $0.02(-0.06$ to 0.10$)$ & 0.64 & $0.28(0.18$ to 0.39$)$ & $<0.001$ \\
\hline Triglycerides (mg/dL) & $0.08(0.00$ to 0.16$)$ & $<0.05$ & $0.22(0.11$ to 0.32$)$ & $<0.001$ \\
\hline HDL-C (mg/dL) & $-0.07(-0.15$ to 0.02$)$ & 0.11 & $-0.03(-0.14$ to 0.08$)$ & 0.59 \\
\hline LDL-C (mg/dL) & $0.00(-0.08$ to 0.08$)$ & 0.96 & $0.26(0.15$ to 0.37$)$ & $<0.001$ \\
\hline eGFR $\left(\mathrm{mL} / \mathrm{min} / 1.73 \mathrm{~m}^{2}\right)$ & $-0.28(-0.36$ to -0.20$)$ & $<0.001$ & $-0.35(-0.45$ to -0.24$)$ & $<0.001$ \\
\hline Alcohol consumption (g/week) & $0.02(-0.06$ to 0.10$)$ & 0.64 & $-0.05(-0.16$ to 0.06$)$ & 0.35 \\
\hline Current smoker & $0.01(-0.07$ to 0.09$)$ & 0.77 & $-0.15(-0.26$ to -0.04$)$ & $<0.01$ \\
\hline Regular exerciser & $0.02(-0.06$ to 0.10$)$ & 0.67 & $0.05(-0.06$ to 0.16$)$ & 0.35 \\
\hline Fatty liver & $0.07(-0.02$ to 0.15$)$ & 0.11 & $0.25(0.14$ to 0.35$)$ & $<0.001$ \\
\hline Postmenopausal state & - & - & 0.43 (0.33 to 0.52$)$ & $<0.001$ \\
\hline
\end{tabular}

baPWV, brachial-ankle pulse wave velocity; BMI, body mass index; eGFR, estimated glomerular filtration rate; GGT, $\gamma$-glutamyltranspeptidase; HDL-C, high-density lipoprotein cholesterol; LDL-C, low-density lipoprotein cholesterol.

state did not greatly alter the association (model 3). Thus, $\log _{2}$ GGT was significantly associated with baPWV independently of covariates in women $(\beta=0.11,95 \% \mathrm{CI}$ 0.02 to $0.19, \mathrm{p}<0.05)$, but not in men $(\beta=-0.04,95 \%$ CI -0.10 to $0.03, \mathrm{p}=0.28$; model 3 ).

\section{DISCUSSION}

We examined the association between arterial stiffness measured by baPWV and GGT in the Japanese population. The present cross-sectional study clearly indicated that $\log _{2}$ GGT significantly correlated with the baPWV level independent of age, BMI, the volume of consumed alcohol and the presence of fatty liver in women, but not in men. Our data were in agreement with the results of a previous study ${ }^{14}$ showing that serum GGT was associated with baPWV only in women.

GGT is the enzyme responsible for the extracellular catabolism of glutathione. ${ }^{27}$ GGT is found not only in the liver but also in other organ tissues, including the kidney, lung, pancreas and vascular endothelium. ${ }^{1}$ The hydrolysis of glutathione originates cysteinyl-glycine, which is a powerful reductant of $\mathrm{Fe}^{3+}$, able to simultaneously generate $\mathrm{Fe}^{2+}$ and a free thiyl radical. Thereafter, oxygen reactive species, by the same reaction, lead to LDL oxidation and most likely contribute to other processes of atherosclerosis, such as metalloproteinase activation, cell proliferation and apoptosis. ${ }^{28}$ In fact, catalytically active GGT has been found within the atherosclerotic cerebral carotid from autoptic studies and surgical endoarterectomy, co-localised with oxidised LDLs and CD68+ foam cells. ${ }^{29-31}$ It suggests that the pathogenetic action of GGT may involve its localisation inside the arterial wall.

Elevated serum GGT might indicate an increment of oxidative stress at the vascular endothelium. Expression of GGT gene and certain mRNA subtypes are increased during oxidative stress in the inflammatory atheroma within the vascular endothelial wall, ${ }^{32}$ and produced GGT protein acts as a strong reducing agent of the superoxide ion and hydrogen peroxide. ${ }^{1}$ In fact, one isoform of the GGT protein detected in the supernatant of plaque homogenates was comparable with serum GGT and a significant correlation was observed between plaque GGT deposits and corresponding GGT levels. $^{31}$ Those observations may indicate that elevated serum GGT includes GGT proteins produced in the plaque corresponding to oxidative stress. GGT proteins produced in the endothelium cell may have a significant effect on GGT levels, because the total weight of the endothelium cell of the whole body would be equal to that of the liver. ${ }^{33}$

We considered that one possible mechanism of sexual discrepancy regarding GGT with baPWV was the difference in sexual hormones, especially oestradiol. In women, oestradiol produced by the granulosa cells of the ovaries is one of the steroid hormones and protects cells from the oxidative stress-induced cell. ${ }^{34}$ Although small amounts of oestradiol are produced in men by the adrenal cortex and the testis, ${ }^{35}$ oestradiol levels in men are roughly comparable to those in postmenopausal women. Oestradiol has various antiatherogenic effects mediated by different mechanisms, for example, rapid vasodilatory effect via release of nitric oxide, inhibition of smooth-muscle cell proliferation ${ }^{36}$ and protective effect on oxidative stress. ${ }^{37}$ However, the antiatherogenic effects of oestradiol are thought to be reduced along with the attenuation of oestradiol in perimenopausal women. For that reason, some atherogenic mechanisms including oxidative stress may activate gradually along with the reduction of oestradiol.

Furthermore, GGT levels might be influenced by oestradiol levels. ${ }^{34}$ GGT levels are low in premenopausal 
Table 3 Multivariable linear regression analyses for baPWV in males and females

\begin{tabular}{|c|c|c|c|c|c|c|}
\hline & \multicolumn{2}{|l|}{ Model 1} & \multicolumn{2}{|l|}{ Model 2} & \multicolumn{2}{|l|}{ Model 3} \\
\hline & $\overline{\beta, 95 \% ~ C l ~(l o w ~ t o ~ h i g h) ~}$ & p Value & $\overline{\beta, 95 \%} \mathrm{Cl}$ (low to high) & p Value & $\overline{\beta, 95 \% ~ C l ~(l o w ~ t o ~ h i g h) ~}$ & p Value \\
\hline \multicolumn{7}{|l|}{ Males } \\
\hline $\log _{2}$ GGT (IU/L) & 0.08 (0.01 to 0.15$)$ & $<0.05$ & $-0.03(-0.09$ to 0.04$)$ & 0.43 & $-0.04(-0.10$ to 0.03$)$ & 0.28 \\
\hline Age (years) & $0.43(0.36$ to 0.49$)$ & $<0.001$ & $0.38(0.32$ to 0.45$)$ & $<0.001$ & $0.40(0.34$ to 0.47$)$ & $<0.001$ \\
\hline BMI $\left(\mathrm{kg} / \mathrm{m}^{2}\right)$ & ND & - & $-0.12(-0.18$ to -0.05$)$ & $<0.001$ & $-0.14(-0.21$ to -0.07$)$ & $<0.001$ \\
\hline Systolic blood pressure $(\mathrm{mm} \mathrm{Hg})$ & ND & - & $0.51(0.45$ to 0.57$)$ & $<0.001$ & $0.52(0.45$ to 0.58$)$ & $<0.001$ \\
\hline Fasting plasma glucose (mg/dL) & ND & - & $0.11(0.05$ to 0.17$)$ & $<0.001$ & $0.10(0.04$ to 0.16$)$ & $<0.01$ \\
\hline Triglycerides $(\mathrm{mg} / \mathrm{dL})$ & ND & - & $0.03(-0.03$ to 0.10$)$ & 0.29 & $0.02(-0.04$ to 0.08$)$ & 0.54 \\
\hline eGFR $\left(\mathrm{mL} / \mathrm{min} / 1.73 \mathrm{~m}^{2}\right)$ & ND & - & $-0.05(-0.11$ to 0.02$)$ & 0.14 & $-0.05(-0.12$ to 0.01$)$ & 0.12 \\
\hline Current smoker & ND & - & ND & - & 0.09 (0.03 to 0.15$)$ & $<0.01$ \\
\hline Regular exerciser & ND & - & ND & - & $-0.04(-0.10,0.02)$ & 0.22 \\
\hline Fatty liver & ND & - & ND & - & $0.06(-0.01,0.13)$ & 0.11 \\
\hline \multicolumn{7}{|l|}{ Females } \\
\hline $\log _{2}$ GGT (IU/L) & $0.19(0.10$ to 0.28$)$ & $<0.001$ & 0.11 (0.03 to 0.20$)$ & $<0.05$ & 0.11 (0.02 to 0.19$)$ & $<0.05$ \\
\hline Age (years) & 0.53 (0.44 to 0.62$)$ & $<0.001$ & $0.41(0.32$ to 0.50$)$ & $<0.001$ & $0.32(0.20$ to 0.45$)$ & $<0.001$ \\
\hline BMI $\left(\mathrm{kg} / \mathrm{m}^{2}\right)$ & ND & - & $-0.14(-0.24$ to -0.05$)$ & $<0.01$ & $-0.16(-0.25$ to -0.06$)$ & $<0.01$ \\
\hline Systolic blood pressure $(\mathrm{mm} \mathrm{Hg})$ & ND & - & $0.38(0.29$ to 0.47$)$ & $<0.001$ & $0.38(0.29$ to 0.47$)$ & $<0.001$ \\
\hline Fasting plasma glucose (mg/dL) & ND & - & $0.04(-0.04$ to 0.13$)$ & 0.34 & $0.03(-0.05$ to 0.11$)$ & 0.47 \\
\hline Triglycerides $(\mathrm{mg} / \mathrm{dL})$ & ND & - & $0.07(-0.02$ to 0.16$)$ & 0.11 & $0.03(-0.06$ to 0.12$)$ & 0.52 \\
\hline eGFR $\left(\mathrm{mL} / \mathrm{min} / 1.73 \mathrm{~m}^{2}\right)$ & ND & - & $-0.10(-0.19$ to -0.02$)$ & $<0.05$ & $-0.11(-0.19$ to -0.02$)$ & $<0.05$ \\
\hline Current smoker & ND & - & ND & - & $0.01(-0.07$ to 0.08$)$ & 0.89 \\
\hline Regular exerciser & ND & - & ND & - & $-0.04(-0.12$ to 0.04$)$ & 0.29 \\
\hline Fatty liver & ND & - & ND & - & $0.09(-0.01$ to 0.18$)$ & 0.07 \\
\hline Postmenopausal state & ND & - & ND & - & $0.06(-0.05$ to 0.18$)$ & 0.28 \\
\hline
\end{tabular}

Model 1 is adjusted for age.

Model 2 includes model 1 plus BMI, systolic blood pressure and biochemical data (fasting plasma glucose, triglycerides, eGFR).

Model 3 includes all variables in model 2 plus parameters of lifestyles (current smoker (yes), regular exerciser (yes)), fatty liver (yes) and postmenopausal state (yes).

baPWV, brachial-ankle pulse wave velocity; BMI, body mass index; eGFR, estimated glomerular filtration rate; GGT, $\gamma$-glutamyltranspeptidase; ND, Not done. 
women and GGT levels in men are roughly comparable with those of postmenopausal females. ${ }^{38}$ Recent clinical studies regarding the hormone replacement therapy have indicated the favourable effect of oestradiol on GGT levels, ${ }^{38-40}$ and even in the physiological menstrual cycle, GGT levels go up and down along with the oestradiol level. ${ }^{34}$ Since elevated serum GGT might reflect both of the activated atherogenic mechanisms and the reduction of oestradiol, it can be said that GGT is a marker for oestradiol and oxidative stress. For the above reasons, GGT might be significantly correlated with baPWV independent of other atherogenic factors only in women. So when we observe elevated serum GGT in women, especially premenopausal women, we should check them using some screening tests for atherosclerosis including baPWV.

Some limitations of our study should be noted. First, the cross-sectional nature of our study does not permit the determination of causality. Second, this study included only Japanese participants, and therefore these findings may not be generalisable to other biogeographic ethnic groups. Lastly, we did not measure the serum oestradiol levels, so we could not assess the relationship between GGT and oestradiol in this study.

\section{CONCLUSIONS}

We demonstrated a significant correlation between GGT levels and baPWV in Japanese women. When we observe elevated serum GGT in women, especially premenopausal women, we should survey atherosclerosis using a noninvasive test such as baPWV, in addition to performing hepatic tests including abdominal ultrasonography and autoantibodies as indicators for autoimmune hepatitis.

Acknowledgements The authors thank all the staff members in the Medical Health Checkup Center at Murakami Memorial hospital.

Contributors TF contributed to the conception and design, drafting of the manuscript, and analysis and interpretation of the data. $\mathrm{MH}$ contributed to the conception and design, critical revision of the manuscript, analysis and interpretation of the data and approved the final version of the submitted manuscript. TKo, YO, AO and TKa contributed to the acquisition of the data. NN and MF contributed to the critical revision of the manuscript.

Funding This work was supported by the Young Scientists (B) from Japan Society for the Promotion of Science, No.23790791.

Competing interests None.

Patient consent Obtained.

Ethics approval The Medical Health Checkup Center at Murakami Memorial Hospital, Gifu, Japan.

Provenance and peer review Not commissioned; externally peer reviewed.

Data sharing statement The technical appendix, statistical code and data set are available from Masahide Hamaguchi at the Dryad repository, which will provide a permanent, citable and open access home for the data set. doi:10.5061/dryad.m484p.

Open Access This is an Open Access article distributed in accordance with the Creative Commons Attribution Non Commercial (CC BY-NC 4.0) license, which permits others to distribute, remix, adapt, build upon this work noncommercially, and license their derivative works on different terms, provided the original work is properly cited and the use is non-commercial. See: http:// creativecommons.org/licenses/by-nc/4.0/

\section{REFERENCES}

1. Mason JE, Starke RD, Van Kirk JE. Gamma-glutamyl transferase: a novel cardiovascular risk biomarker. Prev Cardiol 2010;13:36-41.

2. Lim J-S, Yang J-H, Chun B-Y, et al. Is serum $\gamma$-glutamyltransferase inversely associated with serum antioxidants as a marker of oxidative stress? Free Radic Biol Med 2004;37:1018-23.

3. Lum G, Gambino SR. Serum gamma-glutamyl transpeptidase activity as an indicator of disease of liver, pancreas, or bone. Clin Chem 1972;18:358-62.

4. Kazemi-Shirazi L, Endler G, Winkler S, et al. Gamma glutamyltransferase and long-term survival: is it just the liver? Clin Chem 2007;53:940-6.

5. Ruttmann E, Brant LJ, Concin H, et al. Gamma-glutamyltransferase as a risk factor for cardiovascular disease mortality: an epidemiological investigation in a cohort of 163,944 Austrian adults. Circulation 2005;112:2130-7.

6. Wannamethee SG, Lennon L, Shaper AG. The value of gamma-glutamyltransferase in cardiovascular risk prediction in men without diagnosed cardiovascular disease or diabetes. Atherosclerosis 2008;201:168-75.

7. Loomba R, Doycheva I, Bettencourt R, et al. Serum $\gamma$-glutamyltranspeptidase predicts all-cause, cardiovascular and liver mortality in older adults. J Clin Exp Hepatol 2013;3:4-11.

8. Fraser A, Harris R, Sattar N, et al. Gamma-glutamyltransferase is associated with incident vascular events independently of alcohol intake: analysis of the British Women's Heart and Health Study and Meta-Analysis. Arterioscler Thromb Vasc Biol 2007;27:2729-35.

9. Hamaguchi M, Kojima T, Takeda N, et al. Nonalcoholic fatty liver disease is a novel predictor of cardiovascular disease. World $J$ Gastroenterol 2007;13:1579-84.

10. Jung $\mathrm{CH}, \mathrm{Yu} \mathrm{JH}$, Bae SJ, et al. Serum gamma-glutamyltransferase is associated with arterial stiffness in healthy individuals. Clin Endocrinol (Oxf) 2011;75:328-34.

11. Park JS, Kang SA, Yoo JS, et al. Association between $\gamma$-glutamyltransferase, adiponectin and arterial stiffness. $J$ Atheroscler Thromb 2012;19:90-7.

12. Zhu C, Xiong Z, Zheng Z, et al. Association of serum gamma-glutamyltransferase with arterial stiffness in established coronary artery disease. Angiology 2013;64:15-20.

13. Saijo $Y$, Utsugi M, Yoshioka $E$, et al. The relationship of gamma-glutamyltransferase to C-reactive protein and arterial stiffness. Nutr Metab Cardiovasc Dis 2008;18:211-19.

14. Song SH, Kwak IS, Kim YJ, et al. Can gamma-glutamyltransferase be an additional marker of arterial stiffness? Circ $J$ 2007;71:1715-20.

15. Hamaguchi M, Takeda N, Kojima T, et al. Identification of individuals with non-alcoholic fatty liver disease by the diagnostic criteria for the metabolic syndrome. World J Gastroenterol 2012;18:1508-16.

16. Motobe K, Tomiyama H, Koji $\mathrm{Y}$, et al. Cut-off value of the ankle-brachial pressure index at which the accuracy of brachial-ankle pulse wave velocity measurement is diminished. Circ $J$ 2005;69:55-60.

17. Yamashina A, Tomiyama $\mathrm{H}$, Takeda $\mathrm{K}$, et al. Validity, reproducibility, and clinical significance of noninvasive brachial-ankle pulse wave velocity measurement. Hypertens Res 2002;25:359-64.

18. Hamaguchi M, Kojima T, Takeda N, et al. The metabolic syndrome as a predictor of nonalcoholic fatty liver disease. Ann Intern Med 2005;143:722-8.

19. Hamaguchi M, Kojima T, Ohbora A, et al. Protective effect of alcohol consumption for fatty liver but not metabolic syndrome. World $\mathrm{J}$ Gastroenterol 2012;18:156-67.

20. Hamaguchi M, Kojima T, Itoh Y, et al. The severity of ultrasonographic findings in nonalcoholic fatty liver disease reflects the metabolic syndrome and visceral fat accumulation. $A m \mathrm{~J}$ Gastroenterol 2007;102:2708-15.

21. Matsuo S, Imai E, Horio M, et al. Revised equations for estimated GFR from serum creatinine in Japan. Am J Kidney Dis 2009;53:982-92.

22. Suzuki A, Angulo P, St Sauver J, et al. Light to moderate alcohol consumption is associated with lower frequency of hypertransaminasemia. Am J Gastroenterol 2007;102:1912-19.

23. Gunji T, Matsuhashi N, Sato $\mathrm{H}$, et al. Light and moderate alcohol consumption significantly reduces the prevalence of fatty liver in the Japanese male population. Am J Gastroenterol 2009;104:2189-95. 
24. Aaron DJ, Kriska AM, Dearwater SR, et al. Reproducibility and validity of an epidemiologic questionnaire to assess past year physical activity in adolescents. Am J Epidemiol 1995;142:191-201.

25. Ryu S, Chang Y, Kim D-I, et al. Gamma-glutamyltransferase as a predictor of chronic kidney disease in nonhypertensive and nondiabetic Korean men. Clin Chem 2007;53:71-7.

26. Hamaguchi M, Kojima T, Ohbora A, et al. Aging is a risk factor of nonalcoholic fatty liver disease in premenopausal women. World $\mathrm{J}$ Gastroenterol 2012;18:237-43.

27. Paolicchi A, Minotti G, Tonarelli P, et al. Gamma-glutamyl transpeptidase-dependent iron reduction and LDL oxidation-a potential mechanism in atherosclerosis. J Investig Med 1999;47:151-60.

28. Emdin M, Passino C, Donato L, et al. Serum gamma-glutamyltransferase as a risk factor of ischemic stroke might be independent of alcohol consumption. Stroke 2002;33:1163-4.

29. Paolicchi A, Emdin M, Ghliozeni E, et al. Images in cardiovascular medicine. Human atherosclerotic plaques contain gamma-glutamyl transpeptidase enzyme activity. Circulation 2004;109:1440.

30. Franzini M, Corti A, Martinelli B, et al. Gamma-glutamyltransferase activity in human atherosclerotic plaques-biochemical similarities with the circulating enzyme. Atherosclerosis 2009;202:119-27.

31. Zhang H, Forman HJ, Choi J. Gamma-glutamyl transpeptidase in glutathione biosynthesis. Methods Enzymol 2005;401:468-83.

32. Higashi $\mathrm{Y}$, Noma K, Yoshizumi M, et al. Endothelial function and oxidative stress in cardiovascular diseases. Circ J 2009;73: 411-18.
33. Borrás C, Gambini J, Gómez-Cabrera MC, et al. 17 Beta-oestradiol up-regulates longevity-related, antioxidant enzyme expression via the ERK1 and ERK2[MAPK]/NFkappaB cascade. Aging Cell 2005:4:113-18

34. Arredondo $\mathrm{M}$, Núñez $\mathrm{H}$, López $\mathrm{G}$, et al. Influence of estrogens on copper indicators: in vivo and in vitro studies. Biol Trace Elem Res 2010;134:252-64.

35. Carreau S, Lambard S, Delalande C, et al. Aromatase expression and role of estrogens in male gonad: a review. Reprod Biol Endocrinol 2003;1:35

36. Skafar DF, Xu R, Morales J, et al. Clinical review 91: female sex hormones and cardiovascular disease in women. J Clin Endocrinol Metab 1997;82:3913-18.

37. Wassmann S, Baumer AT, Strehlow K, et al. Endothelial dysfunction and oxidative stress during estrogen deficiency in spontaneously hypertensive rats. Circulation 2001;103:435-41.

38. Fletcher CD, Farish E, Dagen MM, et al. Effects of conjugated equine oestrogens with and without the addition of cyclical norgestrel on serum and urine electrolytes, and the biochemical indices of bone metabolism and liver function. Maturitas 1988;9:347-57.

39. Elsheikh M, Hodgson HJ, Wass JA, et al. Hormone replacement therapy may improve hepatic function in women with Turner's syndrome. Clin Endocrinol (Oxf) 2001;55:227-31.

40. McKenzie J, Fisher BM, Jaap AJ, et al. Effects of HRT on liver enzyme levels in women with type 2 diabetes: a randomized placebo-controlled trial. Clin Endocrinol (Oxf) 2006;65:40-4. 